\title{
Observations of Venus near Inferior Conjunction
}

made with the 12 inch equatorial and its ( $3 \frac{1}{4}$ inch) finder.

Bad weather prevented any observations here of the planet Venus at inferior conjunction; the $2^{\text {nd }}, 3^{\text {rd }}$ and $4^{\text {th }}$ of December were cloudy and stormy. It was observed, however, on Nov. $29^{\text {th }}$ and $30^{\text {th }}$ and Dec. $1^{\text {st }}$ and $5^{\text {th }}$.

I enclose two drawings of the planet on two of these dates (Dec. I $^{\text {st }}$ and $5^{\text {th }}$ ) which will show, better than words, what was seen here.

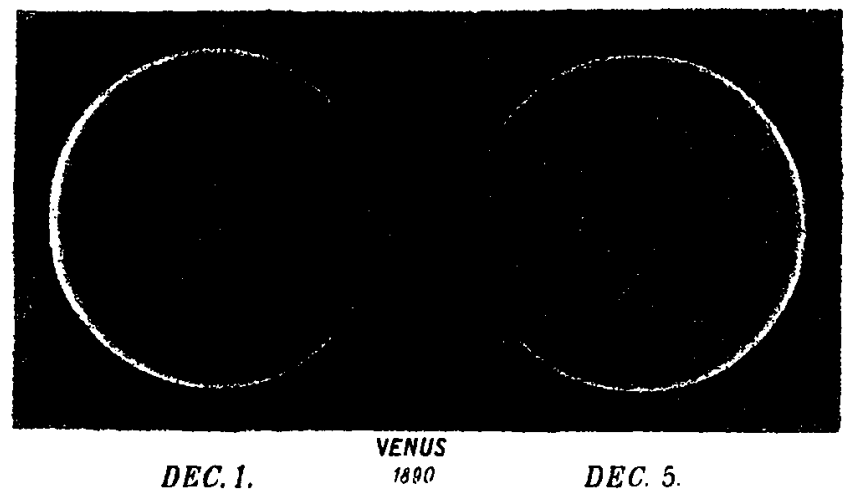

On Nov. 29 the south horn seemed to extend farther than the north and was more slender; this was also the case Dec. ${ }^{\text {st }}$.

Dec. $I^{\text {st }}$ the crescent extends through some $270^{\circ}$, and on Dec. 5 the circle was nearly complete, it could be seen easily through about $340^{\circ}$, and several times $I$ thought it was complete, but our usual poor seing in the daytime made it impossible to be certain of more than I have drawn. I feel sure the complete circle would have been visible if the planet could have been seen on Dec. $4^{\text {th }}$.

No irregularities of the crescent nor markings on the dark body. of Venus could be seen, nor was there any difference of shade to distinguish the dark portion of the planet from the sky.

The following position angles were obtained by placing the wires parallel to the cusps of the crescent.

Nov. $28^{\text {th }}$ position angle of cusps $200: 8$ (4 obs.)

Dec. $I^{\text {st }}>>$, $>8.9(3>)$

All the observations were made about noon.

Mt. Hamilton 1890 Dec. I 2. E. E. Barnard.

\section{Elemente des Planeten (301).}

Die nachstehenden Elemente sind aus 3 Beobachtungen berechnet, welche einen Zeitraum von 27 Tagen unfassen. Der Planet wurde am I6. November 1890 von Herrn Dr. J. Palisa entdeckt und das letzte Mal am I3. December beobachtet.

Epoche : 1890 Nov. 16.5 M. Z. Berlin.

$$
\begin{aligned}
& M=146^{\circ} \quad 6^{\prime} 25^{\prime \prime 8} \\
& \omega=118 \quad 16 \quad 40.6 \\
& \Omega=1422016.0 \\
& i=45249.9 \\
& x=\left[9.9994^{1} 3\right] r . \sin \left(v+350^{\circ} 43^{\prime} 5^{\prime \prime \prime} 8\right) \\
& y=[9.974 \mathrm{I} 90] r \cdot \sin (v+26 \mathrm{I} 4724.2) \\
& z=[9.529826] r \cdot \sin \left(v+25^{2} 2516.3\right)
\end{aligned}
$$

$\varphi=3^{\circ} 4^{\circ} 24^{\prime \prime} .1$

$\mu=788: 373$

$\log a=0.435498$

Aus 2 Beobachtungen Wien Dec. 7 und 8 habe ich einen Normalort gebildet; derselbe wird durch obige Elemente im Sinne $(B-R)$ wie folgt dargestellt :

$$
\mathrm{d} \alpha \cos \delta=-2.2, \quad \mathrm{~d} \delta=+1.7
$$

während der grösste Fehler einer. Beobachtung 2"9 beträgt. Eine handschriftliche Ephemeride wurde Herrn Dr. Palisa von mir bereits mitgetheilt.

Währing I 89 I Jan. 25.

F. Hackenberg.

Vorkaufs-Anzoige. 5 zölliger Reinfelder'scher Refractor ohne Stativ zu verkaufen.

Frau Prof. Teichmüller, Jena.

\section{Inhalt:}

Zu Nr. 3018. H. F. Kiaer. Sur les théries des queues cométaires, 281. - C. F. W. Peters. Eine Bemerkung zum Kepler'schen Problem. 291. E. E. Barnard. On the Nebulosities of the Pleiades and on a New Merope Nebula. 293. $-E$. E. Barnard. Observations of Venus near Inferior Conjunction. 295. - Ұ. Hackenberg. Elemente des Planeten (301). 2 C5. - Verkaufs-Anzeige. 295.

Geschlossen r8gi Febr. 5. Hlcrausgeber: A. K rueger. Druck vou C. S ohaidt. Expedition: Sternwarte in Riel. 
Astr. Nachr. Bd.126. M. Wolf. Phofogr. Stemhelligkeifen.
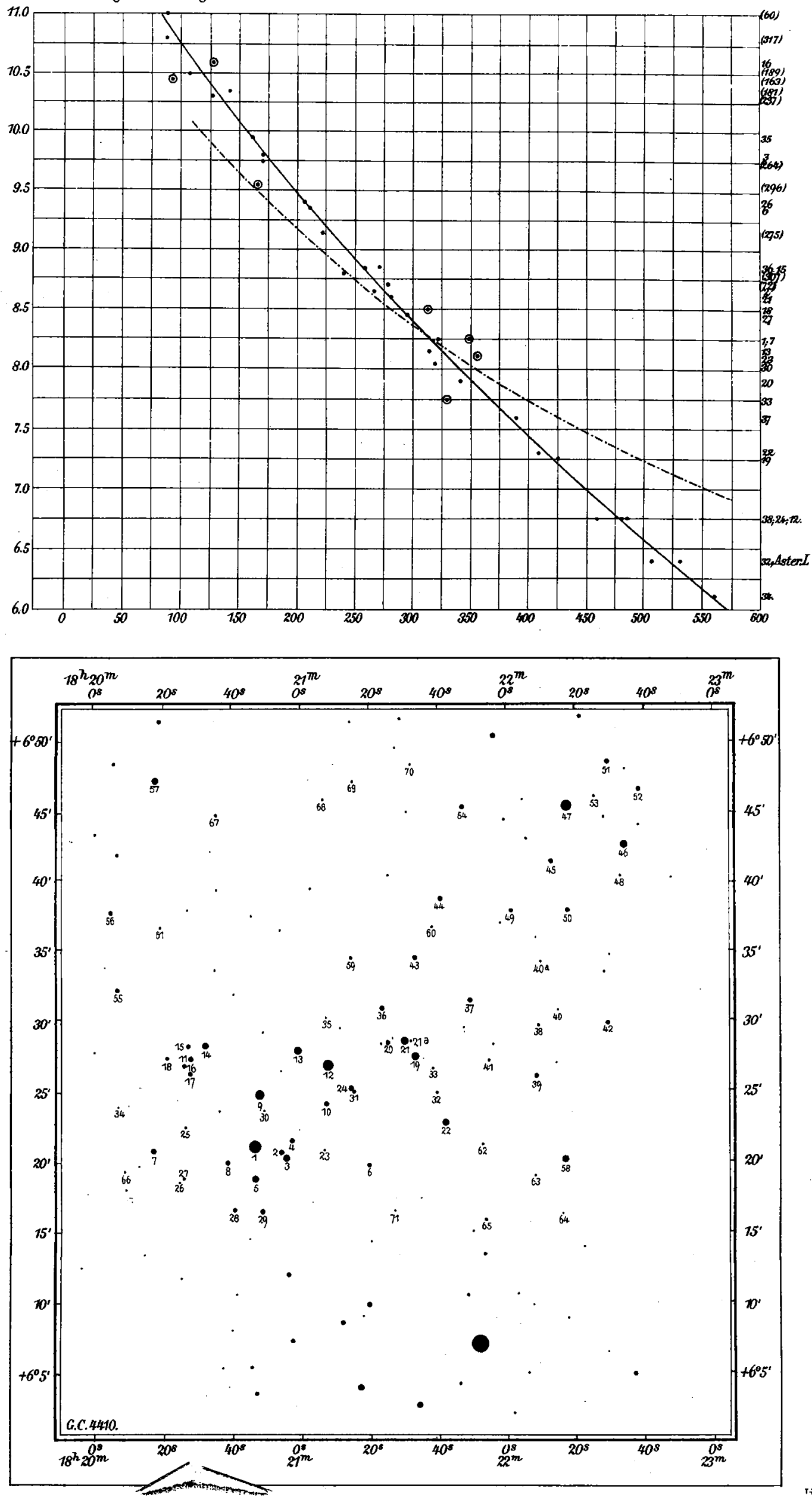